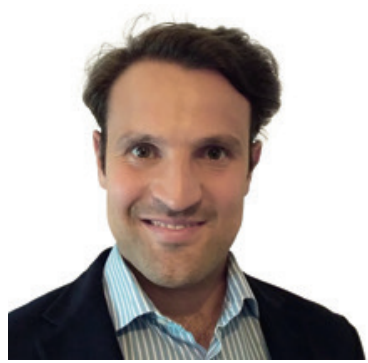

\title{
Diabetic macular oedema: structural and functional results of sequential dexamethasone and aflibercept therapy after one year of treatment
}

Emiliano Di Carlo

Augenklinik, Städtische Klinikum Karlsruhe, Karlsruhe, Germany

Abstract of Hernández-Bel L, Cervera-Taulet E, Navarro-Palop C, et al.: Sequential dexamethasone and aflibercept treatment in patients with diabetic macular edema: structural and functional outcomes at 52 weeks. Ophthalmologica 2019;241:98-104.

\section{Keywords}

Diabetic macular edema . Aflibercept - Dexamethasone implant

\begin{abstract}
Objective: The aim of this paper is to compare intravitreous aflibercept versus dexamethasone implant followed by aflibercept (sequential treatment group) in patients with diabetic macular edema (DME).
\end{abstract}

Methods: We conducted an observational retrospective study in naïve DME patients, 15 treated only with aflibercept (a monthly injection for the first 5 consecutive doses, followed by an injection every 2 months), and 15 treated with a single dexamethasone implant followed by bimonthly aflibercept. Best-corrected visual acuity (BCVA), central macular thickness (CMT), and qualitative features as well as adverse events were assessed at baseline and at 2,6, and 12 months.
Results: BCVA increased from $70.8 \pm 4.1$ to $83.5 \pm 2.7$ letters with aflibercept and from $75.6 \pm 2.7$ to $86.5 \pm 2.5$ with sequential treatment $(p=0.551)$. CMT decreased from $411 \pm 26.1$ to $288.1 \pm 10.5$ with aflibercept and from $411.4 \pm 24.3$ to $260.8 \pm 17.9$ in the sequential treatment group. The differences between the 2 groups, in terms of visual gain and decreased MT, were not statistically significant $(p>0.05)$. Nine and 6 injections and 9 and 7 monitoring visits were performed.

Conclusion: Sequential treatment in DME, starting with dexamethasone and followed by aflibercept, is a promising alternative that can reduce the treatment burden in the first year without statistically significant differences in terms of visual gain and decreased MT compared to aflibercept only.

(c) 2018 S. Karger AG, Basel

Karger ${ }^{\prime \prime}=$ 


\section{Knowledge transfer}

\section{Background}

Diabetic macular oedema (DMO) is the most common cause of blindness in patients with diabetes mellitus, with a prevalence of $7 \%$ [1]. In recent years, two fundamentally different therapeutic strategies - treatment with anti-VEGF and treatment with corticosteroids - have been intensively investigated.

Several randomised clinical studies have proven the benefit of the administration of anti-VEGF drugs with regard to best corrected visual acuity (BCVA) and also to the reduction in central retinal thickness (CRT) [2-4]. The intravitreal dexamethasone implant was approved in July 2014 by the European Medicines Agency (EMA) for the treatment of patients with visual acuity reduction as a result of $\mathrm{DMO}[5]$.

The objective of the study discussed here was to investigate the visual acuity results of the treatment-naive DMO patients. Two therapeutic approaches were compared: intravitreal dexamethasone implant, followed by monthly injections of aflibercept, versus treatment with a standardised aflibercept protocol.
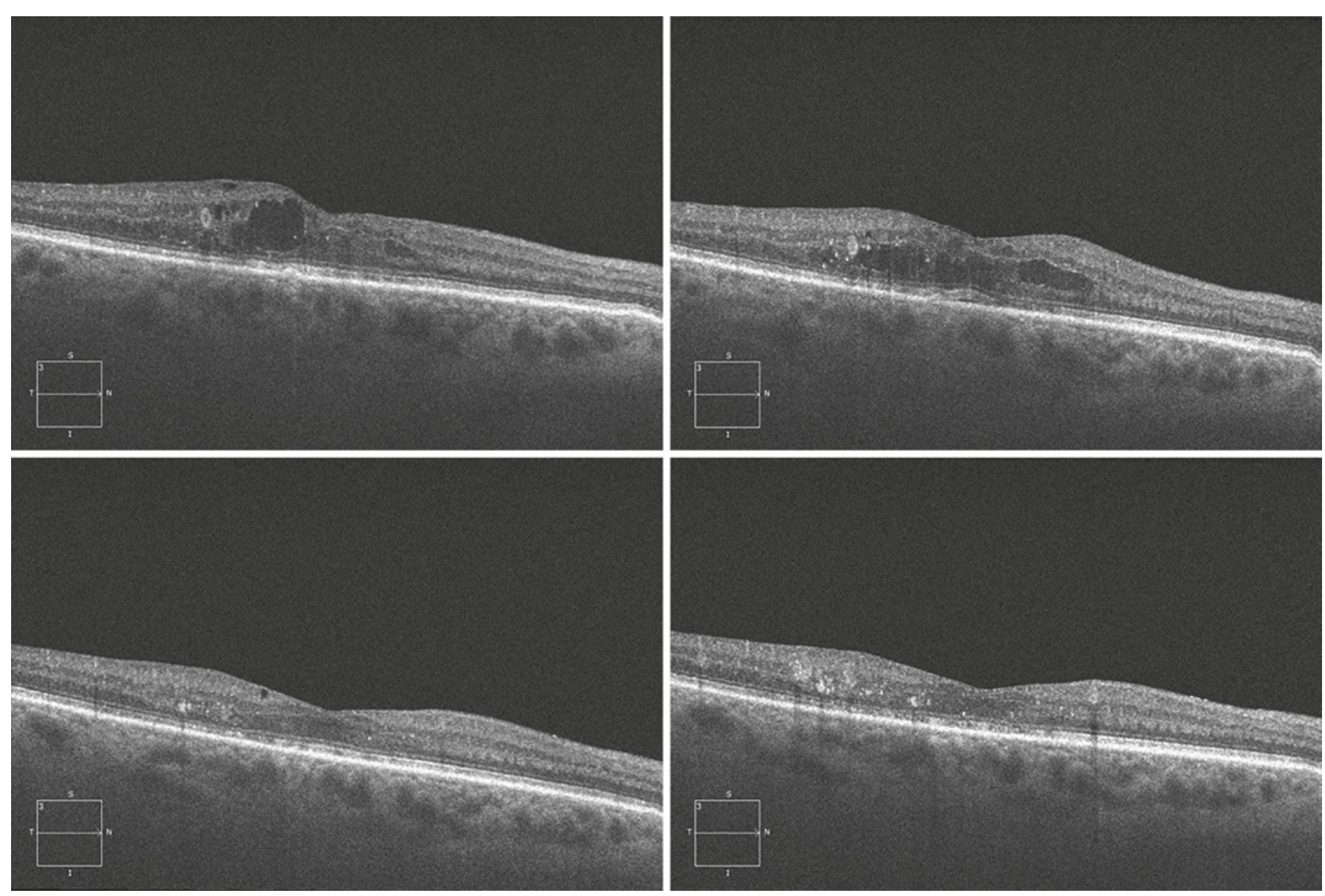

Fig. 1. Our experience with combined (sequential) therapy with dexamethasone and aflibercept: SD-OCT examinations (start of treatment, 2 months, 6 months, 12 months). Resolution of the SRF and improvement in intraretinal cysts.

\section{Critique}

The optimal treatment of DMO represents a clinically relevant challenge. Various studies indicate that the combination treatment consisting of anti-VEGF and intravitreal steroid administration can achieve an earlier improvement in visual acuity and a longer dura-

This is a retrospective observational study of patients with the initial gnosis of DMO who were treated within a year (follow-up periweeks).

Group 1 received a standardised treatment protocol (5 monthly lowed by bimonthly intravitreal aflibercept injections starting from the 16th week.

BCVA and CRT were compared between the two groups at the end of treatment (52 weeks). No statistically significant difference was observed. 
tion of effect $[6,7]$. In this connection, the sequential therapy offers the option of combining the effects of an anti-VEGF therapy, such as the inhibition of vascular permeability and angiogenesis, with the anti-inflammatory effect of the cortisone (stabilisation of the blood-retina barrier, the regulation of the activity of the Müller cells, among other things). In this way, the advantages of both therapeutic strategies can be used.

The need for frequent repeated treatment in the case of anti-VEGF monotherapy - in spite of the positive effects - has numerous disadvantages which ultimately often minimise the advantages of this strategy: in particular, the significant burden on the patients and their family members as well as costs for the healthcare system must be mentioned here. The former often leads to reduced compliance, which in turn diminishes the results.

For this reason, the standardisation of sequential treatment, starting with a dexamethasone implant and followed by aflibercept injections, can reduce the burden of treatment, especially in the first year, by reducing the number of doctor visits and also the costs for the healthcare system (Fig. 1).

\section{Conclusion}

The sequential treatment modality, which consists of a dexamethasone implant and aflibercept, represents an important alternative treatment method in DMO patients. In clinical practice, the stringent treatment intervals which are necessary in anti-VEGF monotherapy are often not adhered to since patient compliance decreases and thus, the ultimately necessary treatment costs can even increase. Therefore, the combination of a sequential dexamethasone treatment with aflibercept provides physicians as well as patients with a good remedy for the treatment of this chronic disease which requires many years of continuous therapy.

\section{Disclosure Statement}

I hereby declare that there are no conflicts of interest with regard to this commentary.

\section{Republication}

This article was first published in Karger Kompass Ophthalmol 2019; 5:163-165.

\section{References}

1 Yau JW, Rogers SL, Kawasaki R, et al.; Meta-Analysis for Eye Disease (METAEYE) Study Group. Global prevalence and major risk factors of diabetic retinopathy. Diabetes Care 2012;35:556-564.

2 Cheung N, Mitchell P, Wong TY: Diabetic retinopathy. Lancet 2010;376:124136.

3 Diabetic Retinopathy Clinical Research Network, Elman MJ, Aiello LP, et al.: DRCR.net. Protocol I: randomized trial evaluating ranibizumab plus prompt or deferred laser or triamcinolone plus prompt laser for diabetic macular edema. Ophthalmology 2010;117:1064-1077.

4 Elman MJ, Bressler NM, Qin H, et al.: Diabetic Retinopathy Clinical Research Network. Expanded 2-year follow-up of ranibizumab plus prompt or deferred laser or triamcinolone plus prompt laser for diabetic macular edema. Ophthalmology 2011;118:609-614.

5 Schmidt-Erfurth U, Garcia-Arumi J, Bandello F, et al.: guidelines for the management of diabetic macular edema by the European Society of Retina Specialists (EURETINA). Ophthalmologica 2017;237:185-222.

6 Faghihi H, Roohipoor R, Mohammadi SF, et al.: Intravitreal bevacizumab versus combined bevacizumab-triamcinolone versus macular laser photocoagulation in diabetic macular edema. Eur J Ophthalmol 2008;18:941-948.

7 Ahmadieh H, Ramezani A, Shoeibi N, et al.: Intravitreal bevacizumab with or without triamcinolone for refractory diabetic macular edema; a placebo controlled, randomized clinical trial. Graefes Arch Clin Exp Ophthalmol 2008;246:483-489.

Kontaktadresse: Dr. Emiliano Di Carlo, Augenklinik, Städtisches Klinikum Karlsruhe, Moltkestraße 90, 76133 Karlsruhe, Germany, emi.dicarlo@ hotmail.it 